\title{
Non-Destructive Elemental Analysis of High-Value Artifacts With Micro-XRF
}

\author{
Andrew H. Lee, Tara Nylese
}

Edax Inc., 91 McKee Drive, Mahwah, NJ 07430 USA

Analysis of inorganic elements in archaeological artifacts is commonly used to determine vital properties of the item, such as where, when, and how it was made. Micro X-ray Fluorescence (micro$\mathrm{XRF}$ ) is a non-destructive technique that can provide qualitative, quantitative, and distributional information from the sample, with minimal sample preparation. With potentially high-value artifacts, this is extremely beneficial and important in maintaining the integrity of the sample.

Micro-XRF is an elemental technique which uses an X-ray tube to generate Brehmsstrahlung radiation, which is then either internally reflected using a glass capillary, or mechanically collimated. This allows beam diameters to range from as low as $\sim 20 \mu \mathrm{m}$, up to $2 \mathrm{~mm}$, while still retaining high count-rates. Based on the intensities of the fluoresced elemental lines radiating off the sample and into the detector, micro$\mathrm{XRF}$ is capable of generating qualitative and quantitative elemental data. In addition, spectrometers include a programmable XYZ stage to move the sample underneath the X-ray beam, allowing elemental imaging, or mapping.

As mentioned, one of the benefits of micro-XRF is the minimal amount of sample preparation it requires, which minimizes damage to the sample. Because an electron beam is not used, typical sample preparation techniques such as polishing and coating the sample are unnecessary. Also, the spot sizes are ideal for the analysis of relatively larger areas, which it may not be possible to analyze with techniques such as scanning electron microscopy. In addition, samples can be run under normal atmospheric pressure, or low vacuum, which provides better sensitivity for lighter elements. As the atomic number increases, the sensitivity improves, making it ideal for inorganic elements.

The following examples illustrate the benefits provided by micro-XRF. Figure 1 shows a segment of parchment (or palimpsest) which was discovered with Byzantine-era artwork. However, it was suspected this palimpsest was an original transcript with text written by Greek mathematician Archimedes, only to be erased and later reused. Here, micro-XRF was able to image the original text by mapping inorganic trace elements from the ink, as shown in Figure 2.

In the second example, micro-XRF was used to image the distribution of elements associated with various pigments on a daguerrotype, an early type of photograph prevalent in the $19^{\text {th }}$ century. Silvercopper plates were exposed to certain chemicals (such as silver, bromine, chlorine) to create a photosensitive surface, which were then processed to create a visible image. By performing an X-ray map, the elemental distribution for different features could be clearly seen, shown in Figures 3-5.

Lastly, a more unique scenario is shown using a sample of lunar rock. If an artifact needs to be protected from environmental conditions, it may be required to analyze the sample with a protective barrier, normally a polymer. To show that micro-XRF can generate a signal strong enough for detection through such a barrier, the sample was covered with polymer films of various composition and thickness, shown in Figure 6. In Figure 7, the resultant Iron map, or $\mathrm{Fe}(\mathrm{K})$, shows that while the polymer will attenuate some escaping energies, the features associated with $\mathrm{Fe}(\mathrm{K})$ are still visible in all regions of the map. 

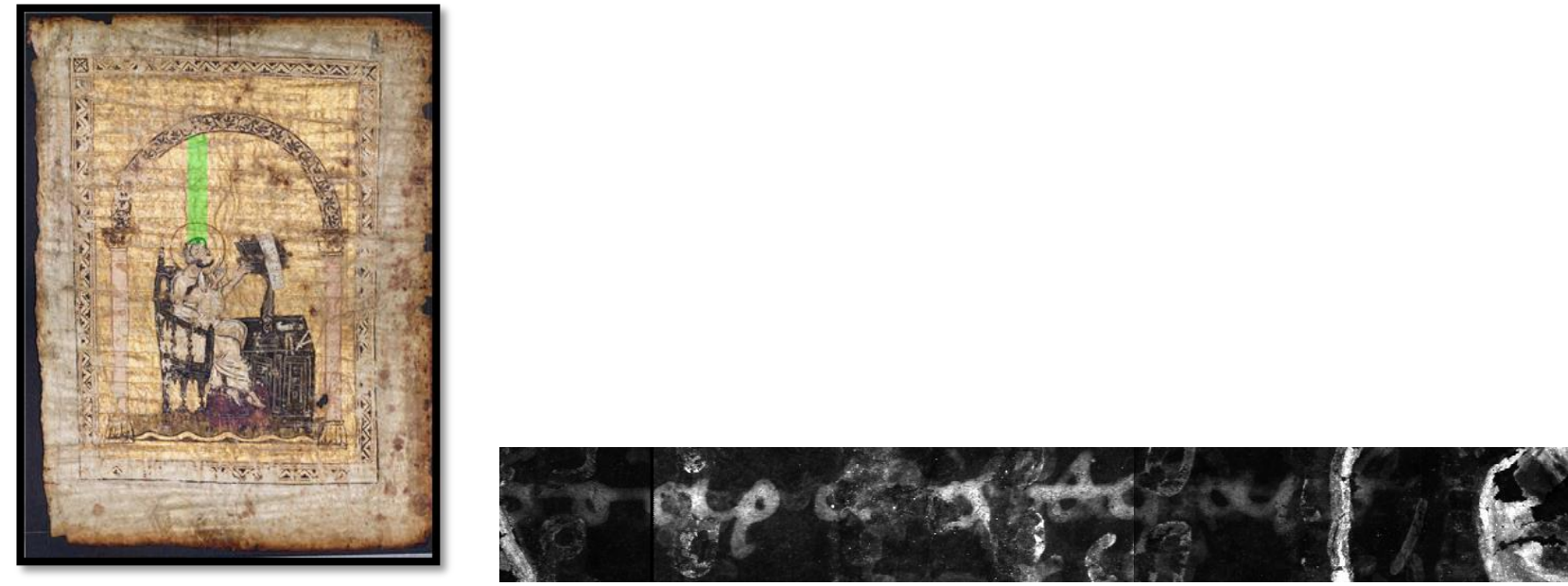

Fig. 1 and 2. Original palimpsest with Byzantine-era art, and grey-scale $\mathrm{Fe}(\mathrm{K})$ map with underlying text.
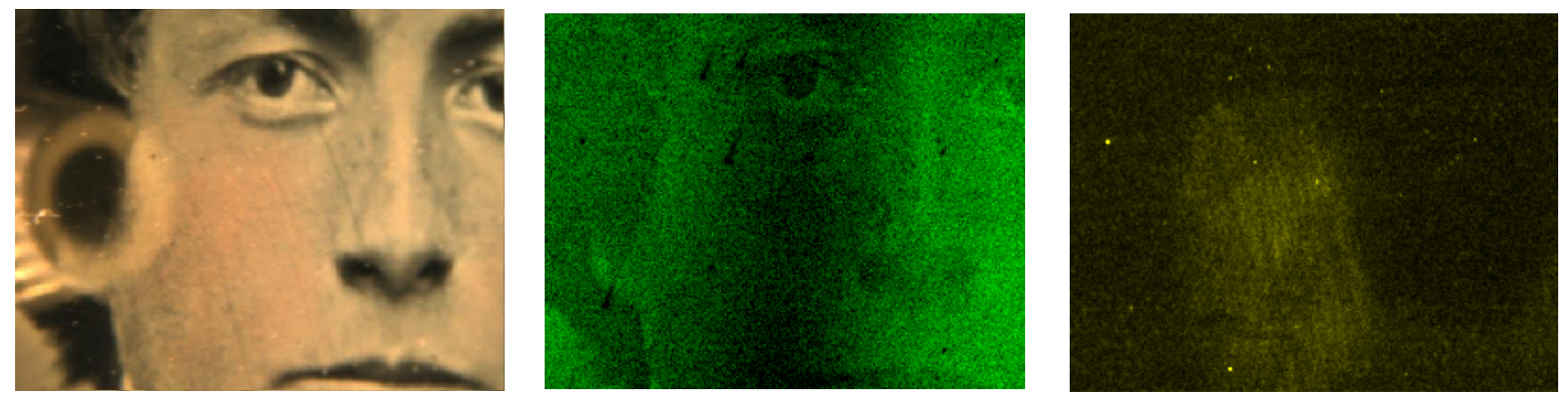

Fig 3. Daguerrotype video image Fig. 4. Gold (Au-L) X-ray map Fig. 5. Iron (Fe-K) X-ray map

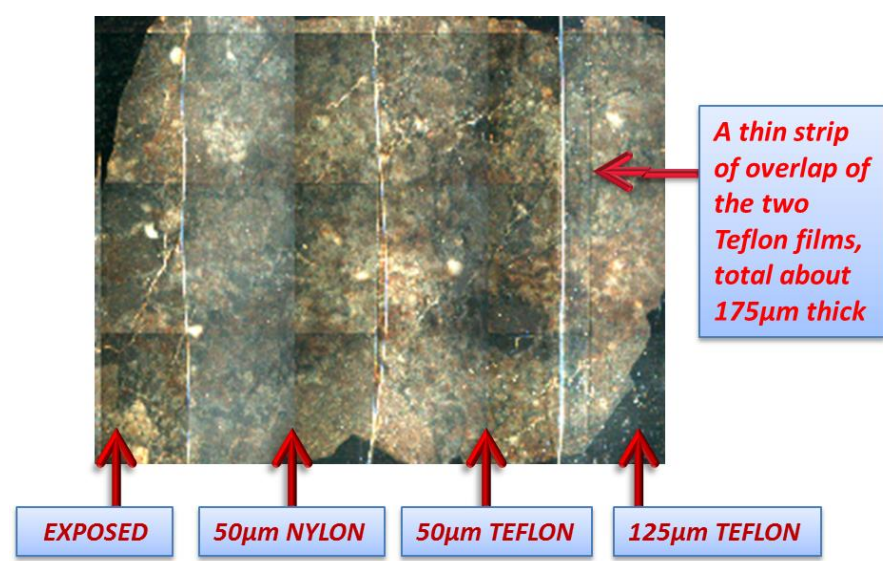

Fig. 6. Image of lunar sample with barrier films

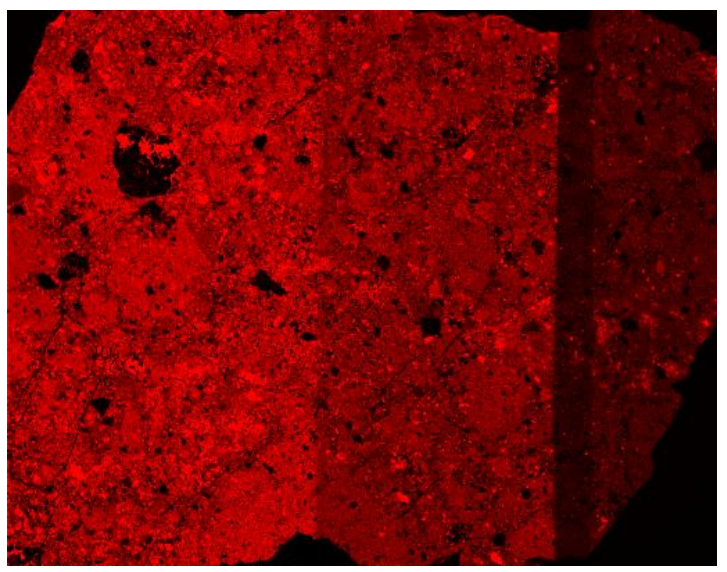

Fig. 7. Resultant $\mathrm{Fe}(\mathrm{K})$ map — Fe(K) energies 